\title{
Developing an individual and collective self-efficacy scale for the teaching of writing in high schools
}

\begin{abstract}
The study reported on here focuses on self-efficacy in relation to high-school teachers' teaching of writing. 140 New Zealand teachers from four schools completed a teacher-ofwriting self-efficacy scale (TWSES) based on a rhetorical model of the writing process and incorporating five hypothesized dimensions. An initial principal components analysis was undertaken on 25 individual self-efficacy items to investigate the dimensionality of the data and the extent to which it reflected the dimensions hypothesized. A twocomponent solution emerged, termed "pre-writing instructional strategies" (accounting for $52 \%$ of total variance) and "compositional strategy demonstration" (7\% of variance). Further principal components analyses conducted on groups of items deemed to be thematically coherent, that loaded on each component, confirmed that the data set for each group, treated separately to any other items, was approximately uni-dimensional. Measurement scales were calibrated to each group of items, and served as the dependent variables for comparisons of teachers' self-efficacy in different subjects. Statistically significant variations occurred in the resultant scale locations for teachers of English, the humanities, science and mathematics. The study findings have implications for the teaching of writing as conceptualized in the secondary school, and indicate a value in viewing disciplinary literacies in rhetorical terms.
\end{abstract}

\section{Keywords}

self-efficacy, teacher efficacy, writing pedagogy, writing across the curriculum 
In the last thirty years, self-efficacy has become a major research focus in studies undertaken from a cognitive or socio-cognitive perspective. Self-efficacy pioneer, Albert Bandura, defined perceived self-efficacy as "concerned with judgments of how well one can execute courses of action required to deal with prospective situations” (1982, p. 122). In other words, self-efficacy is a belief, held either individually or collectively, that future outcomes can be influenced within the context of external constraints, including the discursive constraints associated with disciplinary literacies.

Klassen, Tze, Betts and Gordon (2011) have defined self-efficacy in teachers as "the confidence teachers hold about their individual and collective capability to influence student learning” (p. 21). Over the years, teacher self-efficacy has developed into a twodimensional construct. Following Tschannen-Moran, Woolfolk, Hoy and Hoy (1998), we term the first of these the self-perception of teaching competence, i.e. "the teacher judges personal capabilities such as skills, knowledge, strategies, or personality traits balanced against personal weaknesses or liabilities in this particular teaching context” (p. 228). The second we term the sense of task difficulty, i.e. "the relative importance of factors that make teaching difficult or act as constraints is weighted against an assessment of the resources available that facilitate learning” (p. 228).

The study reported on here focuses on self-efficacy in relation to one aspect of a secondary-school teacher's instructional practice: the teaching of writing. In this respect, it was less concerned with personal teaching efficacy, and more with a specific, but significant, aspect of a teacher's work. 
Out study aim was to develop an instrument to measure teacher-of-writing self-efficacy (TWSES) based on a rhetorical view of the writing process, to analyse its dimensionality and to compare the teacher-of-writing self-efficacy of high-school teachers across a range of subjects. We agreed with Bandura's assertion that: "Scales of perceived self-efficacy must be tailored to the particular domain of functioning that is the object of interest” (2006, pp. 307-8). The rhetorical view of the writing process will be discussed below, and needs to be distinguished from cognitive perspectives developed by such researchers as Flower and Hayes in the early 1980s (Flower \& Hayes, 1981; Hayes, 1996).

This study is timely for a range of reasons. The writing-across-the-curriculum movement has been active in a range of settings for around four decades. More recently, it has merged with a growing focus on, and an attendant literature advocating, the key role of disciplinary literacies (including subject-specific writing) in enhancing student performance across the full range of curriculum areas (see, for example, Mojé, 2008; Shanahan \& Shanahan, 2008). At the same time, there is a widespread recognition in the Anglophone world, of a reluctance for many high-school teachers to view themselves as writers or teachers of writing (Carney \& Indrisano, 2013).

While policy-makers may have much to say on the subject of teacher competence, we view a focus on teacher efficacy in the context of pre-service and in-service teacher education as a far more productive way of approaching professional learning. We would like to think that our efforts to produce a self-efficacy scale in relation to the teaching of 
Developing an individual and collective self-efficacy scale....

writing will contribute to the debate around what this domain-specific construct might look like and how it might be used in research that investigates, for example, the relationship between enhanced self-efficacy around the teaching of writing and student outcomes. (For a modest instance of such an investigation, see Locke, Whitehead \& Dix, 2013.)

The dimensionality of a construct such as a rhetorical understanding of teaching writing can be conceptualised in terms of the number of distinct aspects that underpin it. A construct with two dimensions thus has two distinct aspects, in the sense that individuals vary in their relative strength with respect to each. Constructs such as self-efficacy for the teaching of writing are latent traits, meaning that they can’t be directly measured. Instead, instruments such as questionnaires and surveys are used as proxy measures of these latent traits.

The dimensionality of the data collected using a questionnaire may be investigated using statistical approaches such as principal components analysis or factor analysis. A dimension comprises a subset of items (questions) that, from a qualitative perspective, relate to a common theme, and that, from a statistical perspective, are more highly correlated with one another than they are with other items on the questionnaire.

It is beyond the scope of this article to discuss in detail the relationship between the theorized dimensionality of a construct and the dimensionality of the data arising from an instrument designed to measure it. It should be noted, however, that while questionnaire 
design is usually shaped by a theoretical conception of a construct, the statistical dimensionality of the data collected using that questionnaire often does not reflect the theorized dimensionality of the construct. This proved to be the case in our study. The TWSES was trialled with 140 New Zealand secondary-school teachers. However, the dimensions of self-efficacy we hypothesised in relation to the teaching of writing were drawn from an international literature on the writing process.

\section{A Rhetorical View of the Writing Process}

A number of literature reviews on effective writing instruction have drawn attention to the importance of supporting the writing process and the various strategies associated with it (Hillocks Jr, 2006; Graham \& Perrin, 2007; Myhill, Fisher, Jones \& Lines, 2008). While there is some relationship between theories of writing development and theories around the nature of the writing process (Camp, 2012), our focus here is very much on process - the stages required for a text to be produced in a particular context and the discourses that variously construct this process. We distinguish two understandings of the writing process: a sequence of cognitive operations (associated broadly with psychological theory) and a rhetorical orientation to the production of text (associated with socially situated views of writing).

The view of the writing process as a sequence of cognitive operations, propounded by cognitive psychologists Flower and Hayes $(1980,1981)$, has been highly influential for writing researchers and educational practitioners alike (Vanderburg, 2006). This view focuses on the individual writer and the range and sequencing of tasks required to 
complete the production of a text, from initially conceptualizing the task and its requirements (including some sense of audience and purpose; planning), to locating appropriate content, transforming that content into language fit for task (translating), and finally ensuring that the finished product meets task requirements (reviewing). While this view suggests a linear sequencing of tasks, in fact, writing is very much a recursive (nonlinear) activity. Recursivity refers to the way in which writers are not locked into a set of rigid stages but go backwards and forward between operations such as content generation, translating and revision.

Rhetorical approaches to writing enjoyed a resurgence in the 1990s, through the work of Andrews (1992) and others associated with the new Rhetoric (see Locke, 2015). The writing process as rhetorically oriented views the stages as determined by the contextual demands of a situation involving a rhetor (text-maker or designer), an audience, content, and textual function/purpose. In Bakhtinan terms (1986), it also embraces the concept of intertextuality - texts as utterances in a chain of utterances. Such a view is not solely focused on the individual writer but rather encompasses the social context and the genres and discourses that characterize textual practice in that context. In terms of sequence, there is an overall logic in the steps required in the production of a particular genre, defined by Freedman and Medway (1994) as a "typical way of engaging rhetorically with a recurrent situation” (p. 2). A teacher guiding a student in meeting the requirements of a writing production task will begin with a wide-view consideration of social context, the relationships between participants in this context, and the kinds of textual practice these participants engage in that lead to the emergence of certain types of text or genres. She 
will then move to the study of a particular genre - a process of familiarization - where the novice writer will begin to develop an awareness of the specific requirements for the successful production of the genre. Finally, she will focus on practices that enable the systematic mastery of the skills required to fulfil these requirements, so that when the text is published and disseminated, it fulfills the functions/purposes appropriate to its intended audience.

There is a range of discourses of writing to be found in the literature. Ivanič (2004), for example, distinguishes between "a skills discourse”, “a creativity discourse”, “a process discourse”, "a genre discourse”, “ a social practices discourse” and “a sociopolitical discourse” (p. 225). Locke (2015) distinguishes between cultural heritage, personal growth, rhetorical/textual competence and critical practice discourses of writing. The rhetorical approach to the writing process as schematized in Figure 1 should be thought of as multi-discursive, in that it draws on a range of discourses in the construction of the model, which emphasizes the way writing functions as a socially constructed practice. In relation to our model, we suggest that in understanding and undertaking a writing task, it is helpful for students to think in terms of four levels of consideration. In rhetorical terms, issues related to a particular level can only be addressed when issues related to levels above it have been resolved. For example, issues of tone, at the micro level, can only be resolved through an awareness of the way a topic is being addressed in the wider cultural context, and by audience considerations (how do I best address my audience?). As per Figure 1, these four levels in descending order are: 
Developing an individual and collective self-efficacy scale....

1. Context of culture: defined by Halliday and Hasan as the "broader background against which the text has to be interpreted" (1985, p. 46);

2. Context of situation: the immediate situation that has given rise to the text, intended audience and purpose;

3. Macro features: structure, language function(s), layout, typical content;

4. Micro features: diction, syntax, punctuation and spelling.

Each of these levels poses particular challenges to a writer and requires different writing strategies (and associated skillsets). Because the levels constitute a hierarchy (e.g., 1 needs to be addressed in order to make sense of 2), they suggest both an instructional sequence and also a sequence that individual writers can follow when undertaking a writing task.

[Figure 1 about here]

As discussed in section 3.1, this rhetorical model influenced our development of the teacher-of-writing self-efficacy scale (TWSES).

\section{Self-Efficacy and the Teaching of Writing}

What prompted this study was the first author's involvement in a two-year project entitled "Teachers as writers: Transforming professional identity and classroom practice", which set out, via a series of case studies, to investigate the impact on teachers' self- 
efficacy as writers and teachers of writing of intensive involvement in professional learning based on Writing Workshop principles (see Andrews, 2008; Locke, Whitehead, Dix \& Cawkwell, 2011; Locke, Whitehead \& Dix, 2013). Self-efficacy scales in relation to writing were used with both teacher and student participants in this project, but not self-efficacy scales in relation to the teaching of writing. More recently, the first author has been conducting case studies based in two high schools entitled, “A culture of writing: Impacting on teacher and student performance across the curriculum”. In these projects, the focus shifted to an investigation of ways of building effective communities of writing practice in the secondary-school setting so as to foster teaching/learning processes that enhance writing success for students.

Derived largely from socio-cognitive psychology, self-efficacy rests on a core belief connecting human agency (the way we make choices and exercise control over our lives) and efficacy beliefs. There are a number of reasons why researchers have been interested in measuring self-efficacy. A substantial body of literature indicates a relationship between self-efficacy beliefs in teachers and productive pedagogical practice, as well as a significant impact of self-efficacy on student achievement (Tschannen-Moran et al., 1998, pp. 205, 215, 222). However, Klassen et al. (2011) were more cautious, suggesting in their updated review of the teacher efficacy literature, that there was only "modest empirical support for the theorized connections between teacher efficacy and student outcomes” (p. 38). Indeed, even in respect of research that has demonstrated an association between students' self-efficacy and performance, the statistical relationships demonstrated have usually been correlations (e.g. Pajares, 2003), casting doubt on a 
causal connection between self-efficacy and achievement. A correlation between selfefficacy and achievement might be attributable to higher self-efficacy resulting in higher achievement, to higher achievement resulting in higher self-efficacy, or to higher levels of some other (unmeasured) variable resulting in higher self-efficacy and higher achievement. In general, it is clear that the enhancement of self-efficacy is a means-end project. In the first instance, it is viewed as a means towards increasing such dispositions as motivation and persistence.

In a synthesis of research findings on the relationship between self-efficacy and student writing achievement, Pajares (2003) found that students' self-confidence in relation to their writing capabilities was associated with their motivation to write, as well as with writing performance (typically essay scores). In a study referenced in Pajares’ synthesis, Pajares and Valiante (1997) found that "self-efficacy beliefs made an independent contribution to the prediction of performance" (p. 353) of elementary students' writing as measured by a 30-minute essay-writing task. Another body of literature examines the impact of various interventions on student self-efficacy in writing (see, for example, Schunk \& Swartz, 1993). In the Teachers as writers project, self-efficacy scales in respect of writing were used as pre- and post-intervention measures with both teacher participants in the project (Locke, Whitehead \& Dix, 2013) and students in case-study classes (see Locke \& Kato, 2012).

Most measures of teacher efficacy are not domain- or subject-specific, despite Bandura's original (1982) conception of the self-efficacy construct as being so. More recently, he 
has asserted that, "Scales of perceived self-efficacy must be tailored to the particular domain of functioning that is the object of interest” (Bandura, 2006, p. 308). From our perspective, attempts to develop a writing culture in a high school call for the application of self-efficacy measures across subject areas typical of such a setting. According to Graham, Harris and Fink (2001) studies related to teacher self-efficacy in respect of themselves as teachers of writing are sparse. These researchers, working with primary grade teachers, devised and tested the reliability of an instrument designed to measure self-efficacy in relation to the teaching of writing, and subsequently found that reported classroom practices varied with teachers' levels of self-efficacy, as measured by the scale.

The scale developed by Graham and colleagues was adopted for use with primary teachers from Gibson and Dembo’s (1984) Teacher Efficacy Scale, which contained 30 items using a six-point Likert scale for each statement to indicate respondents' degree of agreement. Gibson and Dembo reported that the scale had two dimensions: Personal teaching efficacy (a teacher's confidence in relation to influencing learning outcomes for their students), and general efficacy (a teacher's confidence in their instructional practices vis-a-vis contextual constraints that might potentially impact negatively on the effectiveness of these practices). Tschannen-Moran et al. (1998) showed that these factors exhibited adequate reliability and were marginally correlated. Graham and colleagues selected from the Gibson and Dembo (1984) instrument 16 items, each of which were significantly and uniquely associated with one of the two dimensions underpinning the scale, and modified them so as to make them applicable to writing instruction in the primary school. An initial, exploratory principal-components analysis of the responses of 
153 primary teachers to the 16 -item scale was conducted. This analysis identified groups of items that were relatively highly correlated with one another, compared with other items in the questionnaire. The analysis was interpreted as showing two principal components, or dimensions, in the data. The first, associated with 10 items, "appeared to reflect teachers' beliefs about their ability to teach writing and affect change in their students”, and the second, associated with six items, “reflected teachers' beliefs concerning limits in the effectiveness of teaching” (p. 189). We view these two dimensions as consistent with the two self-efficacy dimensions we referred to earlier.

Another teacher self-efficacy scale was designed and analysed by Skaalvik and Skaalvik (2007). In this study, the responses of 244 Norwegian primary and middle-school teachers were collected, using a 24-item instrument designed to measure teachers' selfefficacy, using a seven-point Likert scale for each item. Their starting point was an analysis of role expectations in Norwegian schools, which led to a decision to incorporate six subscales in their instrument: "Instruction, Adapting Education to Individual Students' Needs, Motivating Students, Keeping Discipline, Cooperating With Colleagues and Parents, and Coping With Changes and Challenges” (p. 614). Their analysis confirmed other research suggesting that teacher self-efficacy is multidimensional, although they posited a different set of dimensions than Graham et al. (2001). An initial exploratory principal components analysis supported their theoretical model, showing six separate dimensions, each relating to one of the six subscales. A subsequent confirmatory factor analysis supported this interpretation and showed moderate correlations between the six factors. It appears, however, that the confirmatory analysis was carried out using the 
Developing an individual and collective self-efficacy scale....

same data set as the exploratory analysis; the authors do not mention collecting a second sample or sub-dividing the original sample. If this were so, then the confirmation of the dimensionality shown in the exploratory analysis would have been statistically inevitable.

Perceived collective efficacy (PCE) is a construct that has been defined relatively recently and has had far less attention from educational researchers than individual self-efficacy. Perceived collective efficacy was defined by Bandura (1997) as “a group’s shared belief in its conjoint capabilities to organize and execute the courses of action required to produce given levels of attainments” (p. 477). Bandura (2006) suggests that, as a predictor, PCE depends on the extent of "interdependence" in the group, contrasting a team of gymnasts whose success is the sum of individual efforts, and a soccer team which accomplishes its goals on the basis of the way in which players work together. Whatever validity we ascribe to this distinction, it is clear that it cannot be applied easily to a school staff. As Skaalvik and Skaalvik (2007) point out in relation to the Norwegian context, while teachers can be thought of as individuals working within the confines of their own classroom, they are also in various ways working as members of groups and their selfefficacy may well be related to how the group performs.

The increasing interest in PCE in educational research stems from the awareness that no teacher is an island, and that aspects of the cultural context of a school are likely to influence such things as the uptake of certain classroom practices over others, expectations of students, and the ability of staff to address various constraints on potential outcomes (for example, socioeconomic status $\{\mathrm{SES}\}$ ). While relatively few in number, 
some studies have reported connections between PCE, student achievement and individual teacher efficacy. For example, a study by Tschannen-Moran and Barr (2004) found significant positive relationships between teachers’ perceived collective efficacy and student achievement in Grade 8 mathematics, writing and English. In addition, PCE made a significant contribution to students' writing scores independent of their SES. In a later study, Parker, Hannah and Topping (2006) found a significant positive relationship between teachers' perceived collective efficacy (PCE) and reading and writing, with PCE appearing to have a much stronger independent impact than SES on writing (as with Tschannen-Moran \& Barr, 2004). Finally, a study by R. Goddard and Y. Goddard (2001) suggested that PCE is a predictor of teacher self-efficacy.

\section{Method}

\subsection{Instrument}

Our view of writing and writing instruction as a rhetorically oriented process influenced the way we approached the task of developing our teacher-of-writing self-efficacy scale (TWSES) (See Figure 2). As a hypothesis, we identified 5 possible dimensions of teacher-of writing self-efficacy (see Appendix):

1. Orientation competencies: These concerned the relationship of a text with its context, and made use of such concepts as "genre”, "purpose” and "audience”. 
2. Research competencies: These concerned planning and the use of inquiry strategies to generate content appropriate for the particular piece of writing.

3. Structural competencies: These concerned the formulation of a writing plan and concepts such as "structure" and "cohesion".

4. Textural competencies: These concerned concepts such as "voice”, "style”, “vocabulary”, "diction”, "grammar”, and "sentence shapes” (syntax).

5. Motivational competencies: These were concerned with fostering a writing community, using peer response, encouraging goal-setting, using feedback and feed forward, and teacher modeling of a writer identity.

"Orientation Competencies" are viewed as having a reciprocal relationship with contexts of culture and situation. We view context of situation as having two aspects: 1 . the situation which elicits the text; and 2. the classroom context (within the wider context of the school) where the student writer is situated and which has a bearing on how she/he orients her/himself to the task of writing. "Motivational Competencies" recognizes the latter. "Research Competencies" have a reciprocal relationship with the production of textual content (macro features), while "Structural Competencies" also have a reciprocal relationship with macro features, because they deal with the overall unity/coherence of the text. Finally, “Textural Competencies” are viewed as having a reciprocal relationship with micro features, dealing as they do with issues of diction, syntax, punctuation and so on.

[Figure 2 about here] 
In designing the TWSES for use in the study, each item (e.g. OC3: Provide for students a meaningful context and purpose for their writing) requested respondents to choose from four options related to the prompt: "How confident are you that you can? - (1) not confident at all, (2) not very confident, (3) quite confident, and (4) very confident. A fourpoint Likert scale was selected on the basis that it might be difficult for participants to distinguish more than this number of levels of endorsement. No neutral midpoint was included, because it is not clear that a response of neither confident nor unconfident is actually appropriately located between not very confident and quite confident from a psychological point of view. In producing the questionnaire, the randomising function of MS Excel was used to order the 25 items (five for each of the hypothesized dimensions).

In addition to the hypothesized dimensions, the TWSES included seven items related to collective efficacy around the teaching of writing; these were our attempt to articulate a range of broad attributes related to the teaching of writing, and were not based on previous studies. The questionnaire asked respondents to reflect on how well the teachers in their school functioned as a group in relation to the teaching of writing. For items such as "Teachers in this school can motivate even the most difficult pupils to engage in writing” (CE1), respondents chose from a four-point Likert scale: (1) false, (2) mostly false, (3) mostly true, (4) true. Again, a four-point Likert scale was adopted for the reasons given above in relation to individual efficacy. Because the focus of this article is on individual efficacy, we will not be reporting on collective efficacy. 


\subsection{Participants and Procedure}

Participants were 140 New Zealand high-school teachers, drawn from four schools. Three schools were from the Wellington region, and were selected because they reflected a cross-section of school types (co-ed and single-sex, and with varying socio-economic catchment areas). The fourth was an Auckland school participating in the "Culture of writing” project discussed previously. In the case of the Wellington schools, the principals were approached and agreed on the basis of professional goodwill and the assurance that a resultant profile for staff would be provided subsequently. There was no formalized institutional ethics approval process. Rather, completion of the questionnaire itself was regarded as implying the consent of respondents, and a sentence was included in the questionnaire itself to this effect. Completion of the questionnaire was confidential and voluntary. All respondents were asked to indicate their major teaching subject and the number of years they had taught.

In terms of sampling, our approach was purposive in that we sought a range of schools. Because we had little control over questionnaire return rates from Wellington schools, the sampling of teachers was regarded as convenience. In the case of the Auckland school, the return of questionnaires was over $90 \%$ because it was a component of the baseline data for the afore-mentioned project. Returns for the Wellington schools were 24\%, 36\% and $46 \%$ approximately. From our perspective, we saw this as sufficient in terms of the planned data analysis. Conclusions related to analysis in terms of various sub-groups were to be tempered by the size of respective samples. 


\subsection{Data Analysis}

The approach taken to analysis comprised two stages. In the first stage, a principal components analysis was used to identify the dimensionality of the questionnaire data; that is, the number and nature of the constructs represented in the participants' responses, and which items were associated with which constructs. This kind of analysis is typical in work of this kind, although exploratory factor analysis is often used for the same purpose. (In fact, we did also carry out such a factor analysis, which yielded essentially the same results as the principal components analysis.)

Typically in educational research involving questionnaire instruments, 'factor scores' for each participant, on each construct identified in a principal components or factor analysis, are generated for use in further analysis. Alternatively, a simple sum of ordinal Likert values is often calculated across the set of items associated with each construct. Both these approaches have the same problem; they involve treating the ordinal response data as if they were interval data. Instead, therefore, the second stage of our analysis used values resulting from an item-response analysis of the data (Samejima, 1969) to calibrate (interval) measurement variables for the constructs identified in the initial analysis, from the ordinal responses to each of the questions associated with each. Essentially these measurement variables fulfill exactly the same role as the variables generated under more typical procedures, but do not entail the same (false) assumption about the nature of the data. Both the problem associated with the typical procedures, and the use of measurement variables to avoid such problems, are described in more detail below. 
The initial principal components analysis was undertaken on the full set of 25 individual self-efficacy items to investigate the dimensionality of the data and the extent to which it reflected the dimensions (implicitly) hypothesised in the design and grouping of the items. This analysis identified groups of items associated both with common (statistical) dimensions and with common (qualitative) themes. In other words, each dimension identified a group of items that were mutually correlated and which, on inspection, made a meaningful combination based on the content or theme of each of the items comprising the group.

Once the principal components analysis was undertaken and groups of items were identified that satisfied both of the above statistical and qualitative requirements, a calibration exercise was undertaken on each group of items to create a quantitative (measurement) scale for each group. In effect, the calibration exercise provided a means by which the responses of a person to the questionnaire could be transformed into a set (or profile) of scores, where each score corresponds to one of the dimensions identified through the principal components analaysis. For example, if the principal components analysis revealed five dimensions (five groups of items), a person answering the questionnaire would have their responses represented as a profile of five "scale” scores.

The calibration procedure we used is one of a family of such procedures, collectively known as item response analysis. The details of the procedure are well beyond the scope of the present work, but a helpful introductory text is that of Hambleton, Swaminathan 
and Rodgers (1991). The calibration of each group of items onto separate measurement scales drew upon a one-parameter version of Samejima’s (1969) polytomous, graded item response model (see below). As a preliminary step to the calibration, further principal components analyses were conducted to confirm that the data set for each group of items, treated separately from any other items, was uni-dimensional; it is an assumption of the graded-response model that uni-dimensionality is (at least approximately) the case for each scale.

Some explanation of the rationale for, and nature of, the calibration process is in order. A common practice in the social sciences, and in educational research in particular, is to average ordinal category values for each participant from Likert-scale items assumed to measure a property or trait. For example, a four-point Likert scale with categories of Strongly disagree, Disagree, Agree, Strongly agree - these categories establish an order of possible responses - might be coded such that these four categories are assigned values of one, two, three and four respectively. It is common practice then to treat these values as if they were quantitative, by using them to calculate sample means and then conducting statistical tests to compare these means between different groups of respondents.

In fact this practice, while widespread, is quite illegitimate; as noted above, Likert values are ordinal, not interval in nature. When we assign values to them and go on to treat those values as if they were actually quantitative measurement, we are implicitly, and almost always falsely, assuming that the difference in degree of agreement denoted by movement 
from one category to the next is the same for all consecutive pairs of categories. For example, we are assuming that the amount of difference in agreement required to move from Strongly disagree to Disagree is the same as that required to move from Disagree to Agree, because the numerical difference between the values assigned to the former pair of categories (one and two) is the same as that between the values assigned to the latter pair (two and three). The same criticism pertains to the use of factor scores, which are also based on ordinal Likert values.

In contrast, item response models, through the calibration process, locate each endorsement category of each item, as well as each participant, on a truly quantitative (interval) scale, indicating their propensity in respect of each associated theme. Because the measurement scales resulting from the calibration procedure have interval properties, they can be legitimately used as the basis for statistical analyses that compare (using the present study as an example) the mean propensities of different groups of teachers to exhibit self-efficacy in respect of each dimension.

In our work, the scales resulting from each calibration were used as quasi-independent variables in the remaining analyses. More specifically, each calibrated self-efficacy dimension was analysed in relation to teachers' experience (number of years in the profession), and to various disciplines and subject groupings.

\section{Results}


The results of the initial principal components analysis of the individual efficacy data from the questionnaire (Items 1-25) are shown in Table 1. A two-component solution was selected, and items strongly associated, both thematically and statistically, with each of these components, were identified for calibration to measurement scales.

One of the criteria for identifying distinct dimensions in the data using principal components is to identify all components with Eigenvalues greater than one. This means that the component in question accounts for more variability in the data than would be accounted for by individual items, if all items were uncorrelated. In other words, an Eigenvalue greater than one indicates that the items associated with it are mutually correlated.

Although there were three components with Eigenvalues greater than one, the first was very dominant, accounting for $52 \%$ of the total variance of the data. This component was mainly associated with items probing instructional strategies relating to planning a written composition. Six items were identified as being suitable for calibration to a scale related to this theme, labelled Pre-writing instructional strategies: RC10, RC7, OC3, OC2, SC11 and OC4. All of these had strong loadings on Component 1. SC11 also had a substantial loading on component 3, but its loading on Component 1 was much stronger, and component 3 was, in any event, marginal in terms of the proportion of total variance it accounted for. Other items with strong loadings on Component 1 (SC13, SC12 TC17, RC6 and RC8) were excluded from the measure of Pre-writing instructional strategies 
Developing an individual and collective self-efficacy scale....

for various reasons: SC12, SC13 and TC17 have no obvious relationship with instructional strategies; while RC6 and RC 8 are thematically suitable, their loadings on Component 2 were stronger than their loadings on Component 1.

[Table 1 about here]

The second component was much less dominant than the first, accounting for just $7 \%$ of the total variance. Even so, there is thematic consistency amongst the items loading strongly on this component, with most relating to the demonstration of compositional strategies. Again, six items were identified as being suitable for calibration to a scale related to this theme, labeled Compositional strategy demonstration: OC1, OC5, SC15, TC16, RC6 and RC8. All of these items relate to modeling the writing process, and all loaded more strongly on Component 2 than on any other component. While MC21, MC22 and MC23 also loaded strongly on Component 2, none of these relate to demonstrating compositional strategies. MC25 was also excluded. Although it loaded on Component 2 and was thematically suitable, its loading on component 3 was stronger than its loading on Component 2.

Component 3 was deemed not to be a suitable basis for calibrating a measurement variable because its Eigenvalue was only marginally greater than one, being associated with just $6 \%$ of the total variance, because no consistent theme could be identified across the items loading on this component, and because more than half of the items that had substantial association with it were more strongly associated either with component 1 or 
with component 2. Table 2 presents the TWSES items that were retained as a result of this analysis in relation to the two components we settled on.

[Table 2 about here]

Prior to calibrating measurement variables for Pre-writing instructional strategies and Compositional strategy demonstration, two further principal components analyses were conducted on each of the sets of items selected for calibration to these variables. As noted in the data analysis section above, this was done to check the dimensionality of the item sets, it being an assumption of the item response model used for the calibration, that the items to be calibrated relate (approximately) to a single dimension. In both cases, the data for the items to be calibrated showed a single principal component with an Eigenvalue greater than one, with all items loading strongly on this component.

The measurement variables for Pre-writing instructional strategies and Compositional strategy demonstration were moderately, and significantly correlated: $r(138)=.65$, $p<.05$, although neither variable was substantially or significantly correlated with years of teaching experience: $r(136)=.08, p=.35$; and $r(138)=.16, p=.07$. (While the principal components analysis with the orthogonal rotation used here constrains the identified dimensions to be uncorrelated, the measurement variables constructed from the subsets of items associated with each dimension are not so constrained.)

Table 3 shows the mean and standard deviation of the scale locations for self-efficacy in 
Developing an individual and collective self-efficacy scale....

Pre-writing instructional strategies for respondents in each subject group and overall. In many subject groups, the number of respondents was too low to make reliable comparisons with other data. However, four subject groupings: English, the humanities (history, geography, media studies and social studies), science, and mathematics had sufficient respondents to allow for statistical comparisons between the groupings. Of these, the highest mean self-efficacy scale location was for teachers of English, significantly greater than that of humanities teachers: $t(44)=2.13, p=.04$. This in turn was significantly greater than the mean location for science teachers; $t(44)=3.62, p$ $<.001$. There was no significant difference in the scale locations of science and mathematics teachers: $t<1$. It should be noted that the scale units are arbitrary in the sense that negative measurements do not reflect negative self-efficacy (just as negative temperatures on a Celsius scale do not indicate negative heat). Rather, as is evident in Tables 2 and 3, the mean scale location under the calibration method used here is typically close to zero.

[Table 3 about here]

Table 4 shows the mean and standard deviation of the scale locations for self-efficacy in Compositional strategy demonstration for respondents in each subject group and overall. The ordering of subject groupings was similar, although not identical, to those for Prewriting instructional strategies. Of the four most populous groupings, humanities teachers showed the highest mean scale locations, although this was not significantly greater than that of English teachers: $t(46)=1.57, p=.12$. The difference between the 
locations for English and science teachers was marginally greater but still not significant: $t(48)=1.78, p=.08^{1}$, whereas that between science and mathematics teachers was significant: $t(35)=2.64, p=.01$. Aside from the lack of a significant difference between English and humanities teachers, and between Science and English teachers, in selfefficacy for Compositional strategy demonstration, perhaps the most striking difference between the data for the two measurement variables was that, relative to other subject groupings, science teachers appear to be considerably more self-efficacious in respect of Compositional strategy demonstration than of Pre-writing instructional strategies.

[Table 4 about here]

\section{Discussion}

When we designed the questionnaire, we conceptualized five categories (or dimensions) of competency related to the teaching of writing: orientation, research, structure, texture and motivation. As discussed earlier, we based these on a rhetorical view of writing one based on viewing writing instruction as best located in a process that related the text to be written to the contextual demands and constraints of the writing process. While we were interested to see if any of the components/factors to emerge from the questionnaire mirrored these dimensions, we were also interested in what the analysis of these teachers' responses might tell us. While we believed, intuitively, that self-efficacy around the teaching of writing was multi-dimensional, we were open in respect of what these dimensions might be.

\footnotetext{
${ }^{1}$ Traditionally, p should be $\leq .05$ to be called significant.
} 
So what do we make of the results? As Table 1 indicates, the first component identified in the principal components analysis (Pre-writing instructional strategies) indicates a very dominant dimension in relation to self-efficacy around writing instruction. Indeed, the proportion of variance accounted for by Component 1 is seven times that accounted for by Component 2. In relation to the categories we used in creating our TWSES, three items here are Orientation Competencies, three are Research, three are Structural, one is Textural and one (the weakest) is Motivational. Is there something that unifies them? Setting aside MC24, all of the remaining items associated with Component 1 are in various ways related to the way in which a teacher of writing uses a range of strategies to orient their students to the demands of a writing task prior to the commencement of the composition process itself (i.e. putting pen to paper). These strategies include:

- Assisting students in locating content via research (RC10);

- Showing students how to brainstorm and plan (RC7, RC8, SC11);

- Ensuring students have a meaningful purpose and context for their writing (OC3, OC2);

- Helping students to develop their concept of genre and specific genre requirements (OC4, SC12, SC13, TC17);

Because these strategies are linked to the pre-writing stage of the writing process, we have used this term for this dimension of self-efficacy in relation to the teaching of writing. 
The importance of pre-writing (as writing) was highlighted in the cognitive model of the writing process propounded by Flower and Hayes (1981). The component is supported by the substantial reviews of what works in writing instruction cited earlier (Graham \& Perrin, 2007; Myhill et al., 2008), with both reviews drawing attention to the planning stage of the writing process and inquiry activities. Graham and Perrin note that: "Prewriting activities include gathering possible information for a paper through reading or developing a visual representation of their ideas before sitting down to write” (2007, p. 18). There is also support from Myhill et al (2008) for the rhetorical approach to writing instruction which underpinned this study: "A strong argument is put forward in the theoretical literature for the positive potential of using rhetorical approaches to the teaching of writing” (p. 3), though there is also recognition that more research is necessary to ascertain the efficacy of aspects of this approach in practice. Pre-writing in terms of a rhetorical approach includes: making connections between genre and social context; reflecting on the relationship of discipline to genre; constructing a scenario to provide a context of situation for the writing task; and providing an authentic audience for the task text (Locke, 2015).

The items associated with Component 2 (Compositional strategy demonstration) reflect a modestly strong dimension in relation to self-efficacy in relation to writing instruction. The overall mean for this Component 2 across all teachers was higher than for Component 1, perhaps suggesting a greater confidence around or emphasis on teacher demonstration as a practice. The items associated with Component 2 link strategy 
Developing an individual and collective self-efficacy scale....

demonstration with composition, that stage of the writing process, as cognitively

conceptualized, when ideas are being translated into text. The active demonstration of strategies related to composition include:

- Writing alongside students in ways that model writing identity (MC25);

- Building a community of writing practice that includes the modeling of strategies such as peer response (MC22) and goal-setting (MC23);

- Demonstrating to students the specific writing requirements of a particular genre, including research requirements (OC1, OC5, RC6);

- Providing hands-on demonstrations of paragraphing (SC15);

- Facilitating students in developing their own voice (TC16).

The item RC8, "Demonstrate the processes of brainstorming and mind-mapping” was cross-loaded; that is, it displayed associations of similar magnitude with both Component 1 and Component 2. How might this be explained? A general point that must be made to understand this cross-loading is that responders to questionnaire items bring their own understandings to them. While teachers may have a shared understanding of what brainstorming and mind-mapping are, different teachers may think of them in relation to different aspects of the writing process. For example, some teachers may associate mindmapping with pre-writing, where it can be a strategy for planning an investigation. For others, it may be seen as much more closely related to the act of composition, where content is being drawn from the well of long-term memory. In light of the preceding analysis, we have chosen to term this second dimension of self-efficacy in relation to 
writing instruction, "Compositional strategy demonstration".

Finally, to reiterate a point made earlier, we did not consider it appropriate to retain component 3 for further analysis. Nonetheless, we found the items that constitute component 3 interesting because they include all items related to what we called "Motivational competencies" and 4 out of 5 of those we termed "Textural". However, this alone was insufficient to retain the component. While the proportion of variance associated with it was only slightly lower than the proportion associated with component 2, there was no overarching concept to lend coherence to this set of items, and more than half were actually more strongly associated with component 1 or component 2 than they were with component 3.

Our TWSES does not feature "barriers in the item formulations" (Skaalvik \& Skaalvik, 2007, p. 614), despite the recommendation of Bandura (1997). More recently, Bandura reiterated this advice, asserting:

Perceived efficacy should be measured against levels of task demands that represent gradations of challenges or impediments to successful performance. Self-efficacy appraisals reflect the level of difficulty individuals believe they can surmount. If there are no obstacles to overcome, the activity is easily performable and everyone is highly efficacious (2006, p. 311).

We would contend, however, that in a domain-specific task such as the teaching of 
writing in the secondary school context, an argument can be made for distinguishing between challenges inherent in the task itself (in Shulman's [1986] terms, challenges related to professional content knowledge and pedagogical content knowledge) and extrinsic challenges, such as unmotivated students. Our particular interest in designing this study was in the dimensions of professional content knowledge and pedagogical content knowledge related to the teaching of writing, each of which (we have no doubt) posed particular discipline-specific, task-related challenges to the respondents in this study. It is interesting to note, also, that among the examples of self-efficacy scales Bandura himself provided in his 2006 chapter we find one without any obstacles specified (i.e. intrinsic to the task as per the "Driving Self-Efficacy scale” [p. 323]) and one with only some dimensions containing obstacles (“Children's Self-Efficacy Scale” [pp. 326-327]). We have no doubt that there are readers who would want to incorporate items related to general efficacy were they to develop a TWSES of their own.

In broad terms, the subject-related scale locations for components 1 and 2 were not surprising. Like reading pedagogy, the teaching of writing has traditionally been seen (in English-speaking cultures) as the preserve of English teachers, and to a lesser extent, that of teachers of languages other than English and the Humanities. Recent years, however, have witnessed a growing literature emphasizing the importance of disciplinary literacies (including writing) as a key to enhancing student achievement (e.g., Cavagnetto, Hand \& Norton-Meier, 2010; Choi, and Diaz \& Hand, 2010 in science education, and Morgan, 2001 and Bossé \& Faulconer, 2008 in mathematics education.) While science teachers in this New Zealand sample rated relatively highly for Component 2, our data suggest that 
Developing an individual and collective self-efficacy scale....

overall teachers of the traditionally verbal-language-based subjects have substantially higher means on both self-efficacy variables than teachers in other curriculum areas, and that science and mathematics teachers have yet to comfortably identify as teachers of writing.

This last finding is unlikely to be a revelation to researchers working in the area of disciplinary literacies or writing-across-the-curriculum. What it does suggest, of course, is the need for research in ways in which discipline-specific writing instruction can be enhanced, for example, in the sorts of projects described in their review by Norton-Meier, Tippett, Hand, and Yore (2010). Work has begun in our own New Zealand context, by Hitchcock (2012) and by the first author in the two "Culture of writing" projects mentioned earlier.

Let us conclude by making some rather obvious points, which have clear implications for future research. Ivanič’s (2004) work makes it clear that there are multiple versions of what it means to write and (by extension) what it means to teach writing. It is also clear that regardless of how they might like to teach, teachers in all curriculum areas are constrained by policy environments, which construct their subjects in various ways including the kinds of writing to be taught and how these should be assessed. The TWSES we developed was constructed out of our own discursive alignments and responded to by teachers who brought their own histories and discursive beliefs to the table in the act of completing our questionnaire. Naturally, we find ourselves wondering how a significantly large group of teachers sharing somewhat different practice histories 
Developing an individual and collective self-efficacy scale....

and belief systems might have responded to our TWSES. Would the same components have emerged? Perhaps not. In this sense, the factors that have emerged here are discursive creatures of time and place, and therefore likely to be modified in the light of future research revisiting the question of what it really means to teach writing.

\section{References}

Andrews, R. (Ed.) (1992). Rebirth of rhetoric: Essays in language, culture and education. London, UK: Routledge.

Andrews, R. (2008). The case for a National Writing Project for teachers. Reading. CfBT Education Trust.

Bakhtin, M. (1986). The problem with speech genres (V. McGee, Trans.). In C. Emerson \& M. Holquist (Eds.), Speech genres and other late essays: M. M. Bakhtin (pp. 60102). Austin, TX: University of Texas Press.

Bandura, A. (1982). Self-efficacy mechanism in human agency. American Psychologist, 37(2), 122-147.

Bandura, A. (1997). Self-efficacy: The exercise of control. New York, NY: W. H. Freeman.

Bandura, A. (2006). Guide for constructing self-efficacy scales. In F. Pajares \& T. Urdan (Eds.), Self-efficacy beliefs of adolescents (pp. 307-337). Greenwich, CT: Information Age.

Bossé, M., \& Faulconer, J. (2008). Learning and assessing mathematics through reading 
Developing an individual and collective self-efficacy scale....

and writing. School Science and Mathematics, 108(1), 8-17.

Camp, H. (2012). The psychology of writing development - And its implications for assessment. Assessing Writing, 17)(2), 92-105.

Carney, M., \& Indrisano, R. (2013). Disciplinary literacy and pedagogical content knowledge. Journal of Education, 193(3), 39-49.

Cavagnetto, A., Hand, B., \& Norton-Meier, L. (2010). The nature of elementary student science discourse in the context of the science writing heuristic approach. International Journal of Science Education, 32(4), 427-449.

Choi, A., Notebaert, A., Diaz, J., \& Hand, B. (2010). Examining arguments generated by Year 5, 7, and 10 students in science classrooms. Research in Science Education, 40(2), 149-169.

Flower, L., \& Hayes, J. (1980). The cognition of discovery: Defining a rhetorical problem. College Composition and Communication, 31(1), 21-32.

Flower, L., \& Hayes, J. (1981). A cognitive process theory of writing. College Composition and Communication, 32(4), 365-387.

Freedman, A., \& Medway, P. (Eds.). (1994). Learning and teaching genre. Portsmouth, NH: Boynton/Cook.

Hitchcock, D. (2012). Infusing effective literacy practice within secondary school disciplines. (Unpublished doctoral thesis). Curtin University, Perth, Australia.

Gibson, S., \& Dembo, M. (1984). Teacher efficacy: A construct validation. Journal of Educational Psychology, 76(4), 569-582.

Goddard, R. D., \& Goddard, Y. L. (2001). A multilevel analysis of the relationship between teacher and collective efficacy in urban schools. Teacher and Teacher 
Developing an individual and collective self-efficacy scale....

Education, 17(7), 807-818.

Graham, S., Harris, K., \& Fink, B. (2001). Teacher efficacy in writing: A construct validation with primary grade teachers. Scientific Studies of Reading, 5(2), 177-202.

Graham, S., \& Perrin, D. (2007). Writing next: Effective strategies to improve writing of adolescents in middle and high schools - A report to Carnegie Corporation of New York. Washington, DC: Alliance for Excellent Education.

Halliday, M., \& Hasan, R. (1985). Language, context, and text: Aspects of language in a social-semiotic perspective. Geelong, VIC: Deakin University.

Hambleton, R., Swaminathan, R., \& Rodgers, J. (1991). Fundamentals of Item Response Theory. Newbury Park, CA: Sage Publications.

Hayes, J. (1996). A new framework for understanding cognition and affect in writing. In C. Levy \& S. Ransdell (Eds.), The science of writing: Theories, methods, individual differences, and applications (pp. 1-27). Mahwah, NJ: Erlbaum.

Hillocks Jr, G. (2006). Research in writing, secondary school, 1984-2003. L1 Educational Studies in Language and Literature, 6(2), 27-51.

Ivanič, R. (2004). Discourses of writing and learning to write. Language and Education, 18(3), 220-245.

Klassen, R., Tze, V., Betts, S., \& Gordon. K. (2011). Teacher efficacy research 19982009: Signs of progress or unfulfilled promise. Educational Psychological Review, 23, 21-43.

Locke, T. (2015). Developing writing teachers: Practical ways for teacher-writers to transform their classroom practice. New York, NY: Routledge.

Locke, T., Whitehead, D., Dix, S., \& Cawkwell, G. (2011). New Zealand teachers 
Developing an individual and collective self-efficacy scale....

respond to the "National Writing Project” experience. Teacher Development, 15(3), 273-291.

Locke, T., \& Kato, H. (2012). Poetry for the broken-hearted: How a marginal year 12 English class was turned on to writing. English in Australia, 47(1), 61-79.

Locke, T., Whitehead, D., \& Dix, S. (2013). The impact of “Writing Project” professional development on teachers' self-efficacy as writers and teachers of writing. English in Australia, 48(2), 55-69.

Moje, E. (2008). Foregrounding the disciplines in secondary literacy teaching and learning: A call for change. Journal of Adolescent \& Adult Literacy, 42(2), 96-107.

Morgan, C. (2001). The place of pupil writing in learning, teaching and assessing mathematics. In P. Gates (Ed.), Issues in mathematics teaching (pp. 232-244). London, UK: RoutledgeFalmer.

Myhill, D., Fisher, R., Jones, S., \& Lines, H. (2008). Effective ways of teaching complex expression in writing. A literature review of evidence from the secondary school phase (Research Report No. DCSF-RR032). London, UK: The Department for Children, Schools and Families (DCSF).

Norton-Meier, L., Tippett., C., Hand, B., \& Yore, L. (2010). Professional development in teaching disciplinary writing in the context of international science reform efforts. In G. Troia, R. Shankland \& A. Heintz (Eds.), Putting writing research into practice: Applications for teacher professional development (pp. 115-153). New York, NY: The Guilford Press.

Pajares, F. (2003). Self-efficacy beliefs, motivation, and achievement in writing: A review of the literature. Reading \& Writing Quarterly, 19(2), 139-158. 
Developing an individual and collective self-efficacy scale....

Pajares, F., \& Valiante, G. (1997). Influence of self-efficacy on elementary students’ writing. The Journal of Educational Research, 90(6), 353-360.

Parker, K., Hannah, E., \& Topping, K. (2006). Collective teacher efficacy, pupil attainment and socio-economic status in primary schools. Improving Schools, 9(2), 111-129.

Samejima, F. (1969). Estimation of latent ability using a response pattern of graded scores (Psychometric Monograph No. 17). Richmond, VA: Psychometric Society.

Shanahan, T., \& Shanahan, C. (2008). Teaching disciplinary literacy to adolescents: Rethinking content-area literacy. Harvard Educational Review, 78(1), 40-59.

Shulman, L. S. (1986). Those who understand: Knowledge growth in teaching. Educational Researcher, 15(2), 4-14.

Schunk, D., \& Swartz, C. (1993). Goals and progress feedback: Effects on self-efficacy and writing achievement. Contemporary Educational Psychology, 18(2), 337-354.

Skaalvik, E., \& Skaalvik, S. (2007). Dimensions of teacher self-efficacy and relations with strain factors, perceived collective teacher efficacy, and teacher burnout. Journal of Educational Psychology, 99(1), 611-625.

Tschannen-Moran, M., Hoy, A., \& Hoy, W. (1998). Teacher efficacy: Its meaning and measure. Review of Educational Research, 68(2), 202-248.

Tschannen-Moran, M., \& Barr, M. (2004). Fostering student learning: The relationship of collective teacher efficacy and student achievement, leadership and policy in schools. Leadership and Policy in Schools, 3(3), 189-209.

Vanderburg, R. M. (2006). Reviewing research on teaching writing based on Vygotsky’s theories: What we can learn. Reading \& Writing Quarterly, 22(4), 375-393. 


\section{Appendix}

\section{Teacher Self-Efficacy Scale for Writing Instruction}

How confident are you that you can:

\section{Orientation Competencies}

1. Demonstrate to students how to "shape" their writing for a particular audience.

2. Explain to students the concept of writing purpose.

3. Provide for students a meaningful context and purpose for their writing.

4. Explain to students what a genre/text type is.

5. Model to students how to write a genre/text type related to your subject/discipline.

\section{Research Competencies}

6. Model to students the research requirements of a particular type of writing.

7. Explain how different types of writing have different planning requirements.

8. Demonstrate the processes of brainstorming and mind-mapping.

9. Provide where appropriate a logical sequence of research activities related to a writing task.

10. Model to students effective ways of finding resources that "feed" into a particular 
writing task.

\section{Structural Competencies}

11. Model to students how to use research notes in producing a writing plan.

12. Explain to students that different genres/text-types are structured differently.

13. Model to students the writing of each component of a text-type relevant to my subject/discipline.

14. Demonstrate to students how to structure an argument.

15. Model to students cohesive paragraphing.

\section{Textural Competencies}

16. Model to students the relationship between "voice" and style.

17. Explain to students that different genres/text-types require different kinds of vocabulary.

18. Model to students the use and function of different kinds of diction (words).

19. Model to students the use and function of different sentence shapes.

20. Decide when it is appropriate to instruct students in a particular grammar point.

\section{Motivational Competencies}

21. Establish a supportive writing community in my classroom. 
Developing an individual and collective self-efficacy scale....

22. Use peer response in a way that motivates students to learn.

23. Assist students in setting achievable goals related to writing competence.

24. Use feedback and feed forward effectively in relation to student writing.

25. Model a writer "identity" myself as an example to students.

Response categories: (1) not certain at all, (2) somewhat uncertain, (3) quite certain, (4) very certain.

\section{Collective Efficacy}

How well do the teachers in your school function as a group in relation to the teaching of writing?

1. Teachers in this school can motivate even the most difficult pupils to engage in writing.

2. Teachers at this school know how to make writing meaningful for their students.

3. Teachers at this school see themselves as teachers of writing.

4. Teachers at this school relate in a collegial way to share their expertise in relation to the teaching of writing.

5. Teachers at this school share a common philosophy about the teaching of writing across curriculum areas.

6. Teachers at this school know how to tailor their writing instruction to the needs of individual students. 
Developing an individual and collective self-efficacy scale....

7. Teacher at this school are effective in using a range of writing assessment tools to guide their instruction.

Response categories: (1) false, (2) mostly false, (3) mostly true, (4) true. 